\title{
UNA ESPECIE NUEVA DE LA FAMILIA ARTOTROGIDAE (COPEPODA: SIPHONOSTOMATOIDA) DE CUBA
}

\author{
Carlos Varela \\ Acuario Nacional de Cuba (A. N. C.). Calle 1ra \#6002 e/e 60 y 62, C. P. 11300, Playa, La Habana,
} Cuba.varela06@gmail.com

\section{RESUMEN}

Se describe una especie nueva de copépodo perteneciente al género Bradypontius Giesbrecht, 1895, hallado en la región Sur Central de Cuba. Esta es la primera ocasión en que se registra un representante de la familia Artotrogidae Brady, 1880, para el Mar Caribe.

Palabras clave: Copepoda, Siphonostomatoida, Artotrogidae, Bradypontius.

Title: A new species of the family Artotrogidae (Copepoda: Siphonostomatoida) from Cuba.

\section{ABSTRACT}

A new species of copepod belonging to the genus Bradypontius Giesbrecht, 1895, collected in the South Central region of Cuba is described. This is the first record of the family Artotrogidae Brady, 1880, for the Caribbean Sea.

Key words: Copepoda, Siphonostomatoida, Artotrogidae, Bradypontius.

\section{INTRODUCCIÓN}

Los copépodos pertenecientes a la familia Artotrogidae están entre los más primitivos del orden Siphonostomatoida. Muchas de las especies de esta familia han sido halladas asociadas a invertebrados marinos (Johnsson, 2001; Johnsson et al., 2002; Kim, 1996, 1998 y Stock, 1965), aunque la naturaleza de su relación simbiótica con el invertebrado hospedador permanece incierta hasta el momento. Típicamente, las especies de esta familia han sido colectadas en número muy bajo, principalmente de lavados de invertebrados marinos y su hallazgo es poco frecuente (Johnsson y Rocha, 2002; Johnsson y Neves, 2005).

Las especies pertenecientes al género Bradypontius han sido halladas en el Atlántico Norte, la Antártida, el Mediterráneo y el Mar del Japón, por lo que este constituye el primer hallazgo de este género en el Atlántico Occidental Tropical. Material colectado en la región Sur Central de Cuba nos permitió hallar ejemplares de una especie no previamente descrita de este género, la que se presenta a continuación.

\section{OBJETIVO}

- Describir una especie nueva de Copepoda del género Bradypontius para Cuba.

\section{MATERIALES Y MÉTODOS}

Las colectas se efectuaron mediante buceo autónomo (SCUBA). Se tomaron macroalgas del fondo y se introdujeron en una bolsa de polietileno a la que se añadieron gotas de formalina 
y se sacudió el contenido que posteriormente fue tamizado y fijado. El material estudiado se encuentra depositado en el Departamento de Colecciones Naturales Marinas del Acuario Nacional de Cuba.

\author{
RESULTADOS \\ Orden Siphonostomatoida Burmeister, 1835 \\ Familia Artotrogidae Brady, 1880 \\ Género Bradypontius Giesbrecht, 1895 \\ Bradypontius cubensis sp. nov.
}

Figuras 1 y 2

Diagnosis. Cefalosoma más largo que ancho; exopodito de la antena con dos setas; segmento genital al nivel de la abertura genital presenta tres setas, anténula con ocho artejos y rama caudal más ancha que larga.

Diagnosis. Cephalosome longer than wide; exopodite of antena with two setae, genital segment to the level of genital aperture with three setae, antennule with eigth articles and caudal rami wider than long.

Descripción del holotipo. Largo máximo $850 \mu \mathrm{m}(820-910 \mu \mathrm{m})$, excluyendo las setas de la rama caudal, ancho máximo $517 \mu \mathrm{m}$. Proporción entre el largo y el ancho 1.6:1. Cuerpo ciclopiforme, con prosoma de $646 \mu \mathrm{m}$ de largo; cefalosoma alargado cubierto por estructuras semejantes a escamas y con pequeñas setas en sus bordes y epímeros puntiagudos (Fig. 1, A y B). Segmentos del 2 al 4 con epímeros puntiagudos. Urosoma con cinco segmentos. Segmento genital 85:134 $\mu \mathrm{m}$, proporción largo- ancho $0.6: 1$ con una proyección cerca de la abertura genital con tres setas. Tres segmentos postgenitales 26:118 $\mu \mathrm{m}, 32: 96 \mu \mathrm{m}, 64: 85 \mu \mathrm{m}$; proporción longitud: ancho 0.2: 1:0.3:1 y 0.7:1 respectivamente. Rama caudal más ancha que larga, 26:37 $\mu \mathrm{m}$ con seis setas (Fig. 1C).

Anténula con $265 \mu \mathrm{m}$ de largo (sin incluir las setas), con ocho artejos. La longitud de los artejos (medidos a lo largo de su margen posterior) 55, 58, 27, 19, 25, 22, 16 y $44 \mu \mathrm{m}$ respectivamente. Homologías y setación como sigue I-1; II-VIII-8; IX-XIII-2; XIV-1; XVXVI-1; XVII-XVIII-1; XIX-XX-1; XXI-XXVIII-7+ estetasco. Todas las setas lisas. Estetasco en el segmento XXI con $278 \mu \mathrm{m}$ (Fig. 1D).

Antena con $135 \mu \mathrm{m}$, ( $\sin$ incluir la seta distal), con basipodito $40 \mu \mathrm{m}$. Endopodito con dos artejos; artejo $1.26 \mu \mathrm{m}$, desarmado; artejo $2.36 \mu \mathrm{m}$ armado con una seta proximal plumosa, una seta subdistal y dos setas distales, la más corta plumosa, ninguna modificada como una garra. Exopodito de un artejo, con $8 \mu \mathrm{m}$ de largo y con dos setas (Fig. 1E).

Cono oral alargado con $255 \mu \mathrm{m}$ de largo, 0.3 veces la longitud del cuerpo, se curva llegando al final del cefalosoma (Fig. 1F). Mandíbula formada por un estilete con denticiones en su extremo, palpo mandibular ausente (Fig. 1G). Maxílula bilobulada, ambos lóbulos delgados y casi iguales en talla. Lóbulo interno $70 \mu \mathrm{m}$ con una espina corta y una larga seta pinnada; lóbulo externo $75 \mu \mathrm{m}$ armado con una seta pinnada y una seta plumosa (Fig. 1H). Maxila con sincoxa $112 \mu \mathrm{m}$ de largo, garra $159 \mu \mathrm{m}$, curvada distalmente y armada con una seta cerca de su extremo y dos pequeñas espinas en su porción central superior (Fig. 1I). Maxilípedo con cinco artejos, sin coxa con $42 \mu \mathrm{m}$, armada con una seta, basipodito $150 \mu \mathrm{m}$ armado con una seta en su porción subdistal; endopodito con tres artejos; artejo 1 con dos setas; artejo 2 con una seta y artejo 3 con una seta y una garra curvada de $102 \mu \mathrm{m}$ Los artejos miden 42, 34 y 15 de largo respectivamente (Fig. 1J). 

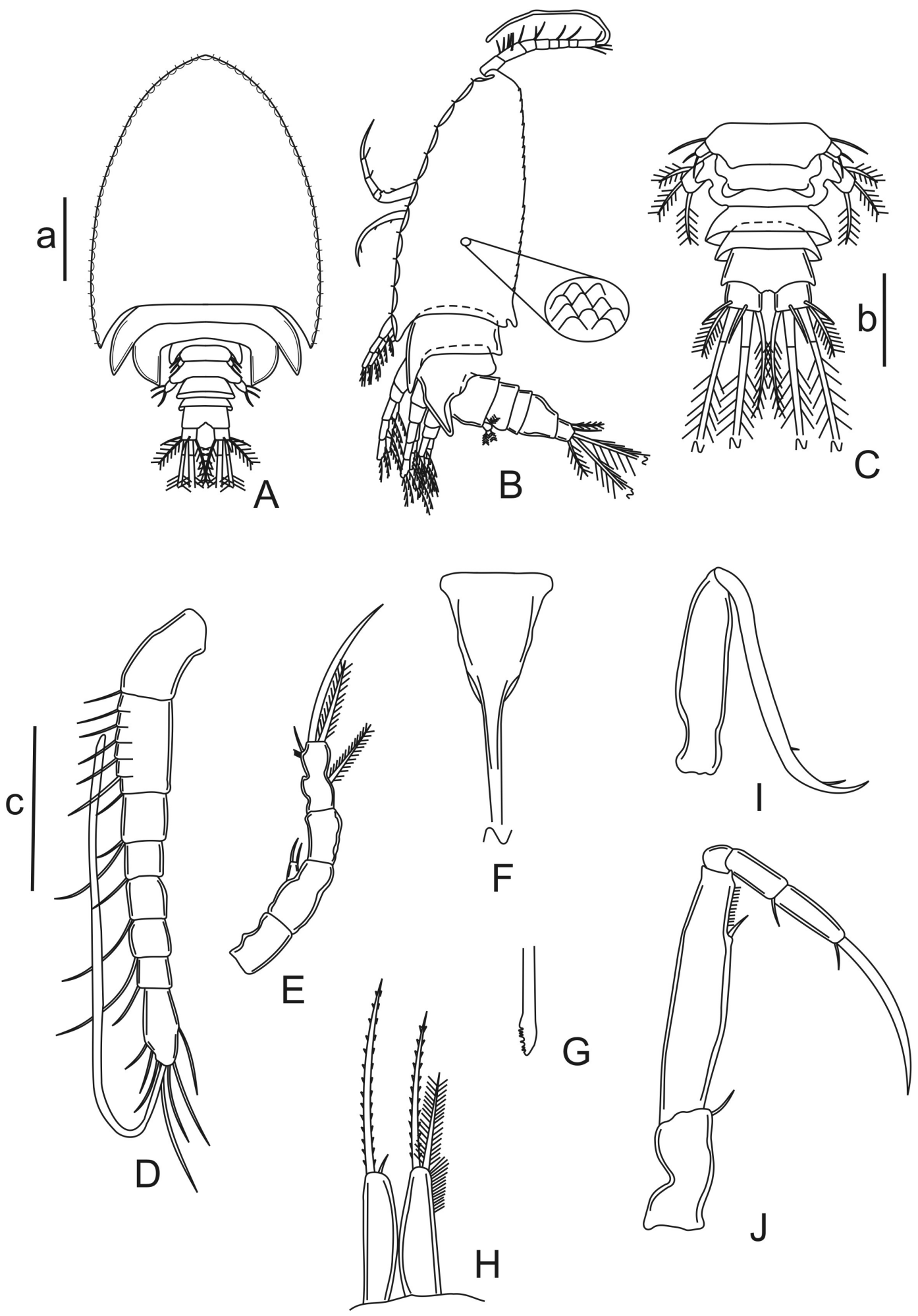

Figura 1. A, vista dorsal del holotipo (a). B, vista lateral del holotipo (a). C, urosoma (b). D, anténula (c). E, antena (c). F, cono oral (c). G, extremo del estilete de la mandíbula (c). H, maxílula (c). I, maxila (c). J, maxilípedo (c). Escala: a, $200 \mu \mathrm{m} ;$ b y c, $100 \mu \mathrm{m}$. 

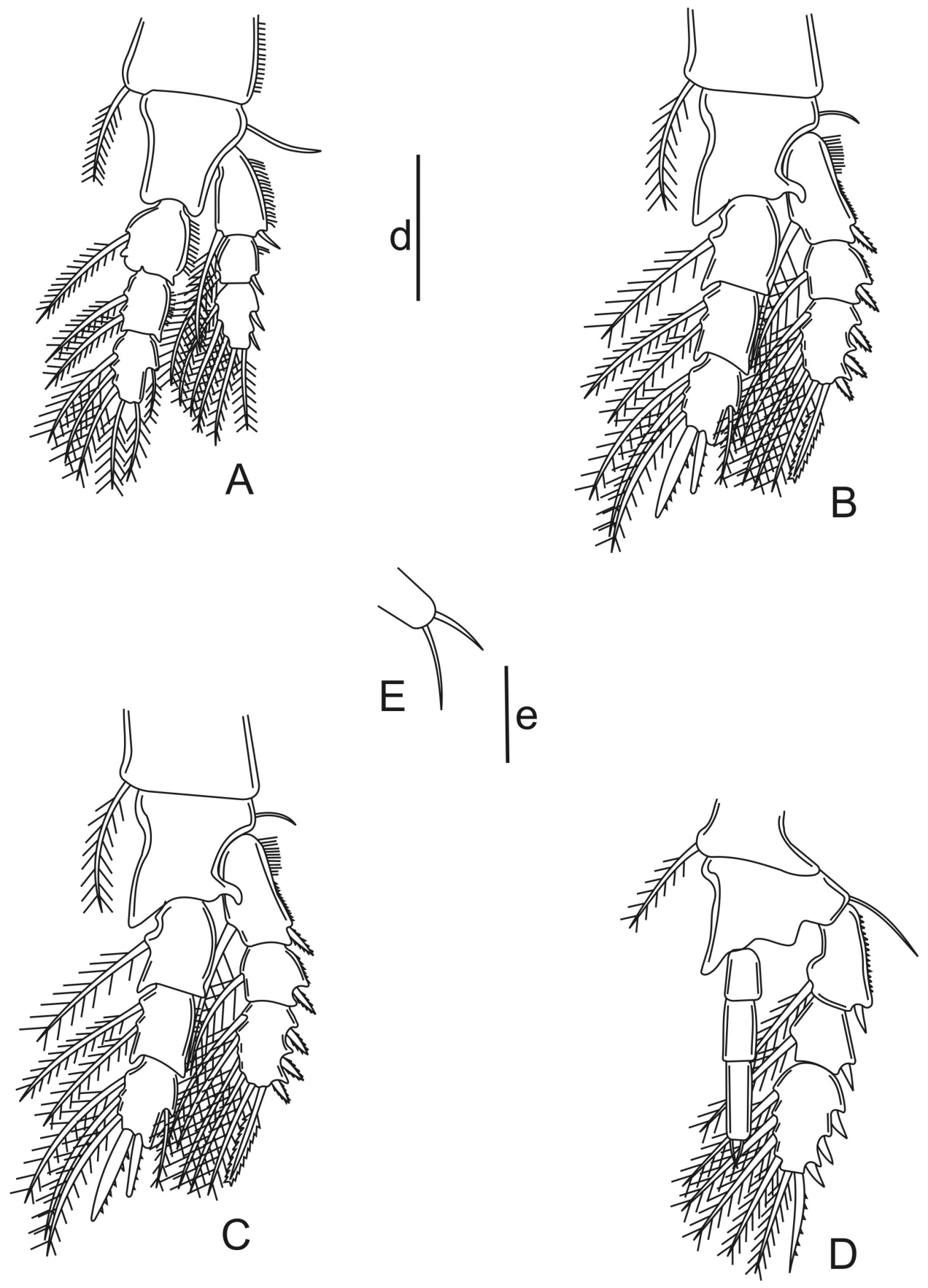
Patas 1 a 4 con dos ramas, cada una con tres artejos (Fig. 2, A-D). La armadura de cada pata se muestra en la Tabla 1.

Tabla 1. Disposición de las espinas y las setas de las patas de Bradypontius cubensis.

\begin{tabular}{|ccccc|}
\hline Pata & Coxopodito & Basipodito & Exopodito & Endopodito \\
\hline P1 & $0-1$ & $1-0$ & I-1; I-1; II,1,4 & $0-1 ; 0-2 ; 1,2,3$ \\
P2 & $0-1$ & $1-0$ & I-1; I-1; III,I,5 & $0-1 ; 0-2 ; 1$, II,3 \\
P3 & $0-1$ & $1-0$ & I-1; I-1; III,I,5 & $0-1 ; 0-2 ; 1$, II,3 \\
P4 & $0-1$ & $1-0$ & I-1; I-1; III,I,5 & $0-0 ; 0-0 ;$ II \\
\hline
\end{tabular}

Pata 5 con el artejo libre armado con 2 setas (Fig. 2E).

Macho. Desconocido.

Etimología. Se denomina cubensis por la isla de Cuba, lugar donde fue colectada esta especie.

Tipos. Holotipo. Hembra no ovígera. CUBA. Colectada en Rancho Luna, provincia de Cienfuegos, 23. iii. 2010 en macroalgas a $20 \mathrm{~m}$ de profundidad; col. C. Varela. Depositado en la Colección Natural Marina del Acuario Nacional de Cuba ANC. 07. 2. 1. 55. Paratipos. Dos hembras no ovígeras. Los mismos datos que el holotipo. ANC. 07. 2. 1. 56.

Comentario. Bradypontius cubensis, especie nueva, presenta la anténula con solo ocho artejos. Dentro del género, solo B. crassisetus Kim, 1996; B. magniceps (Brady, 1880); B. papillatus (Scott, 1888); B. pichoni Stock, 1966 y B. poorei Johnsson, 2002 comparten este carácter. Además, Bradypontius cubensis, especie nueva, presenta la rama caudal más ancha que larga. Carácter este que solo es compartido por B. pichoni. Sin embargo en B. cubensis, especie nueva, el cefalosoma es más largo que ancho, el exopodito de la antena tiene dos setas y el segmento genital al nivel de la abertura genital presenta tres setas; mientras que en $B$. pichoni el cefalosoma es más ancho que largo, el exopodito de la antena tiene tres setas y el segmento genital al nivel de la abertura genital presenta una seta y una espina.

\section{AGRADECIMIENTOS}

A los instructores de buceo Fidel Morejón Alfonso e Israel González Durán del Hotel Rancho Luna, en la provincia de Cienfuegos y a Yenizeys Cabrales Caballero del Centro de Estudios Ambientales de Cienfuegos, por la ayuda en la colecta del material.

\section{LITERATURA CITADA}

Johnsson, R. 2001. Two new artotrogids (Copepoda:Siphonostomatoida) from Madeira Island, Portugal. Hydrobiologia 453/454: 431-440.

Johnsson, R. y C. E. F. Rocha. 2002. Five artotrogids (Crustacea: Copepoda: Siphonostomatoida) From Eastern Antarctica.Memoirs of the Museum of Victoria 59 (2): 439-455.

Johnsson, R., C. E. F. Rocha y C. B. Boyko. 2002. A new species of Cryptopontius (Crustacea: Copepoda: Siphonostomatoida) from Easter Island. American Museum Novitates 3370: 1-8. 
Johnsson, R. y E. Neves. 2005. A revision of Metapontius (Siphonostomatoida: Artotrogidae) with the description of a new species associated with an octocoral from Eniwetok Atoll, Marshall Islands (USA). Zootaxa 1035: 51-59.

Kim, I. H. 1996. Copepoda of Artotrogidae (Siphonostomatoida) from the Sea of Japan. Korean Journal of Systematic Zoology 12 (4): 397-466.

Kim, I. H. 1998. Pulicitrogus compresus gen. nov., sp. nov. (Copepoda, Siphonostomatoida, Artotrogidae) associated with an ascidian in the Sea of Japan. Journal Marine Systems 15: 255-260.

Stock, J. H. 1965. Copepodes associes aux invertebres des cotes du Rousillon. V. Cyclopoides siphonostomes spongicoles rares et nouveaux. Vie Milieu 16: 295-324.

[Recibido: 14 de febrero, 2012. Aceptado para publicación: 12 de mayo, 2012] 